\title{
Association of dopamine-related genetic loci to dopamine D3 receptor antagonist ABT-925 clinical response
}

\author{
A Bhathena, Y Wang, JB Kraft, KB Idler, SJ Abel, RR Holley-Shanks, WZ Robieson, B Spear, L Redden and DA Katz
}

ABT-925, a selective dopamine $D_{3}$ receptor (DRD3) antagonist, was tested in schizophrenia. A DRD3 gene polymorphism results in an S9G amino-acid change that has been associated with lower risk of schizophrenia, higher affinity for dopamine and some antipsychotics, and differential response to some antipsychotics. The effect of S9G genotype on response to ABT-925 was examined. DNA samples $(N=117)$ were collected in a proof-of-concept, double-blind, randomized, placebo-controlled study of ABT-925 (50 or $150 \mathrm{mg} \mathrm{QD)} \mathrm{in} \mathrm{acute} \mathrm{exacerbation} \mathrm{of} \mathrm{schizophrenia.} \mathrm{A} \mathrm{pre-specified} \mathrm{analysis} \mathrm{assessed} \mathrm{impact} \mathrm{of} \mathrm{genotype} \mathrm{(SS}$ versus SG + GG) on change from baseline to final evaluation for the Positive and Negative Syndrome Scale (PANSS) total score using analysis of covariance with genotype, treatment and genotype-by-treatment interaction as factors, and baseline score as covariate. Significant genotype-by-treatment interaction $(P=0.015)$ was observed for change from baseline to final evaluation for the PANSS total score. Within subgroup analyses showed significant improvement from placebo in the SG $+\mathrm{GG}$ group treated with ABT-925 $150 \mathrm{mg}$. More favorable clinical outcomes were observed in patients treated with ABT-925 $150 \mathrm{mg}$ who carried the DRD3 G allele than in those who carried the DRD3 SS genotype.

Translational Psychiatry (2013) 3, e245; doi:10.1038/tp.2013.22; published online 9 April 2013

\section{Introduction}

ABT-925 is a selective dopamine D3 receptor (DRD3) antagonist in vitro. ${ }^{1}$ We recently reported that ABT-925 regimens of 50 and $150 \mathrm{mg}$ QD were well tolerated, but did not significantly improve schizophrenia symptoms in acutely ill patients. ${ }^{2}$ A positron emission tomography study revealed that these regimens resulted in $<60 \%$ receptor occupancy of ABT-925 at the D3 receptor, ${ }^{3}$ suggesting that the hypothesis of D3 receptor antagonism for the treatment of schizophrenia may not have been fully tested. Genetic polymorphisms at dopamine-relevant loci can increase sensitivity to dopamine and dopamine agonists ${ }^{4}$ and may reveal subsets of schizophrenia patients in whom lower doses of D3 receptor antagonists could provide therapeutic benefit.

A single-nucleotide polymorphism in the $D R D 3$ gene results in a greater affinity of DRD3 for dopamine and some D3 receptor antagonists. ${ }^{4}$ Associations between this Ser9Gly (S9G) single-nucleotide polymorphism and response to antipsychotics have been reported, however, treatment effects have been associated with each allele, reviewed in Arranz and de Leon. ${ }^{5}$ A Val158Met (V158M) single-nucleotide polymorphism in the catechol-o-methyl transferase (COMT) gene reduces the ability of COMT to catabolize dopamine, and may affect subjects' response to treatment with antipsychotics. ${ }^{6,7}$ Polymorphisms in the dopamine transporter (SLC6A3) gene have been reported to affect transporter levels in the brain, ${ }^{8}$ potentially modulating the levels of dopamine and affecting the response to antipsychotics. Given the strong evidence of the role of dopamine in the etiology of schizophrenia and the potential of these genetic loci to affect drug response, we examined specific polymorphisms in DRD3, COMT and SLC6A3, and sought to determine whether subjects' genotypes at specific loci were associated with a salutary response to ABT-925.

\section{Materials and methods}

Study design. The clinical trial was a double-blind, randomized, placebo-controlled, escalating-dose, parallel group study conducted in the United States (12 sites), Argentina (3 sites) and Mexico (4 sites). Details of the clinical study (NCT00412620) design and methods are published elsewhere. ${ }^{2}$ Briefly, the study consisted of a screening period (up to 30 days), taper-off/washout (up to 7 days), double-blind (6 weeks) and follow-up (safety monitoring for 5 weeks). A total of 117 acutely ill schizophrenia subjects consented to pharmacogenetic analysis and were included in the subanalysis reported here. Subjects received $50 \mathrm{mg}$ or $150 \mathrm{mg}$ ABT-925 QD or placebo for 6 weeks. The primary efficacy end point was the mean change from baseline to final evaluation in Positive and Negative Syndrome Scale (PANSS) total score. Secondary endpoints included the Negative Symptom Assessment (NSA) and PANSS subscale scores (positive symptoms, negative symptoms and general psychophathology). Analysis of PANSS change including DRD3 genotype as a covariate was a protocol-specified secondary analysis.

Research and Development, AbbVie Inc., North Chicago, IL, USA

Correspondence: Dr A Bhathena, Research and Development, AbbVie Inc., Dept. R4CD, Bldg AP10, 1 North Waukegan Rd, North Chicago, IL 60064-6100, USA. E-mail: anahita.bhathena@abbvie.com

Keywords: ABT-925; clinical response; dopamine D3 receptor; pharmacogenetics; polymorphism; schizophrenia

Received 31 December 2012; accepted 18 February 2013 
Subjects. Independent ethics committees/institutional review boards approved the study protocol, and informed consent was obtained for each subject before any study-related procedures being performed. Male and female subjects 18-65 years old (inclusive) had a current primary diagnosis of schizophrenia or schizoaffective disorder undergoing an acute exacerbation with prominent 'active phase' symptoms, as described by the Diagnostic and Statistical Manual of Mental Disorders, 4th Edition, Text Revision (DSM-IV-TR). For genotype determination, a blood sample was collected from each subject who consented to pharmacogenetic analysis.

Genotyping. Deoxyribonucleic acid (DNA) was isolated from whole blood using the FlexiGene DNA AGF3000 kit (Qiagen, Valencia, CA, USA), on an AutogenFlex 3000 (AutoGen, Holliston, MA, USA). Genotypes at the DRD3313A > G (S9G) and $C O M T$ 417G $>$ A (V158M) single-nucleotide polymorphisms were determined using the pyrosequencing detection method (Qiagen). Primers used for DRD3 S9G PCR were: 5'-ATCACGCACCTCCCTCTGG-3' (forward) and 5'-GCTGG CACCTGTGGAGTTCT-3' (reverse), and the pyrosequencing primer was: 5'-TCTGAGCCAGCTGAGT-3'. Primers used for COMT V158M PCR were: 5'-TCACCATCGAGATCAACCC C-3' (forward) and 5'-CCACAGCCGGCCCTTTT-3' (reverse), and the pyrosequencing primer was: 5'-GGTGGATTTC GCTGG-3'. Genotypes at the SLC6A3 VNTR locus were determined by fragment analysis using a 3130xl Genetic Analyzer and Genemapper 4.0 software for analysis (Applied Biosystems, Foster City, CA, USA). Primers used for SLC6A3 VNTR PCR were: 5'-TGCGGTGTAGGGAACGGCCTGAG-3' (forward) and 5'-CTTCCTGGAGGTCACGGCTCAAGG-3' (reverse). All individuals performing genotyping were blinded to clinical trial data.

Sampling bias. As the subjects could participate in the clinical trial without consenting to pharmacogenetic analysis, sampling bias could have confounded the results of the pharmacogenetic analyses. Sampling bias was assessed using a one-sample test comparing the observed means (or percentages) between the consenting subset to the means (or percentages) of all study subjects. One-sample $t$-tests were performed on subject demographics (age, sex and race), for baseline PANSS total score, and for the change from baseline to the final evaluation for the PANSS total score. The one-sample tests for the percentage of subjects in each treatment group were conducted to compare the mean (or percentage) values of the consenting subgroup to those of the entire study cohort. Each two-sided test was considered statistically significant when $P<0.05$.

Pharmacogenetic analysis for efficacy. To examine if genotype had any impact on subjects' responses to treatment, an analysis of covariance for change from baseline to final evaluation for the PANSS total score was carried out for the intent-to-treat data set (all subjects for whom efficacy assessments were available at baseline and at least one on-treatment visit) with factors for treatment, genotype and interaction term of treatment-by-genotype, and with baseline score as a covariate. This analysis was pre-specified in the protocol for comparison of $D R D 3 \mathrm{SS}$ to $\mathrm{SG}+\mathrm{GG}$ genotypes.
A two-sided test was considered significant when $P<0.05$. Analyses for COMT V158M and SLC6A3 VNTR were exploratory for hypothesis generation. Similar exploratory analyses were completed for the secondary outcome measures, which included the individual subscales of PANSS and NSA-16. Subgroup analyses to compare placebo with each active dose of ABT-925 were performed for the change from baseline to final evaluation for each outcome measure within each genotype group. The baseline score for each outcome measure was used as a covariate. In these analyses, as in the clinical trial analysis, the two placebo groups (placebo for $50 \mathrm{mg}$ ABT-925 and placebo for $150 \mathrm{mg}$ ABT-925) were combined. Qualitative assessment revealed no differences between the groups. ${ }^{2}$

Analyses were performed using JMP Versions 6.0.0, and SAS Version 9.2.0 (both products of SAS Institute, Cary, $\mathrm{NC}$, USA).

\section{Results}

Sampling bias. In all, $67 \%$ of intent-to-treat subjects (117/153) consented for pharmacogenetic research. No statistically significant sampling bias was observed in age,

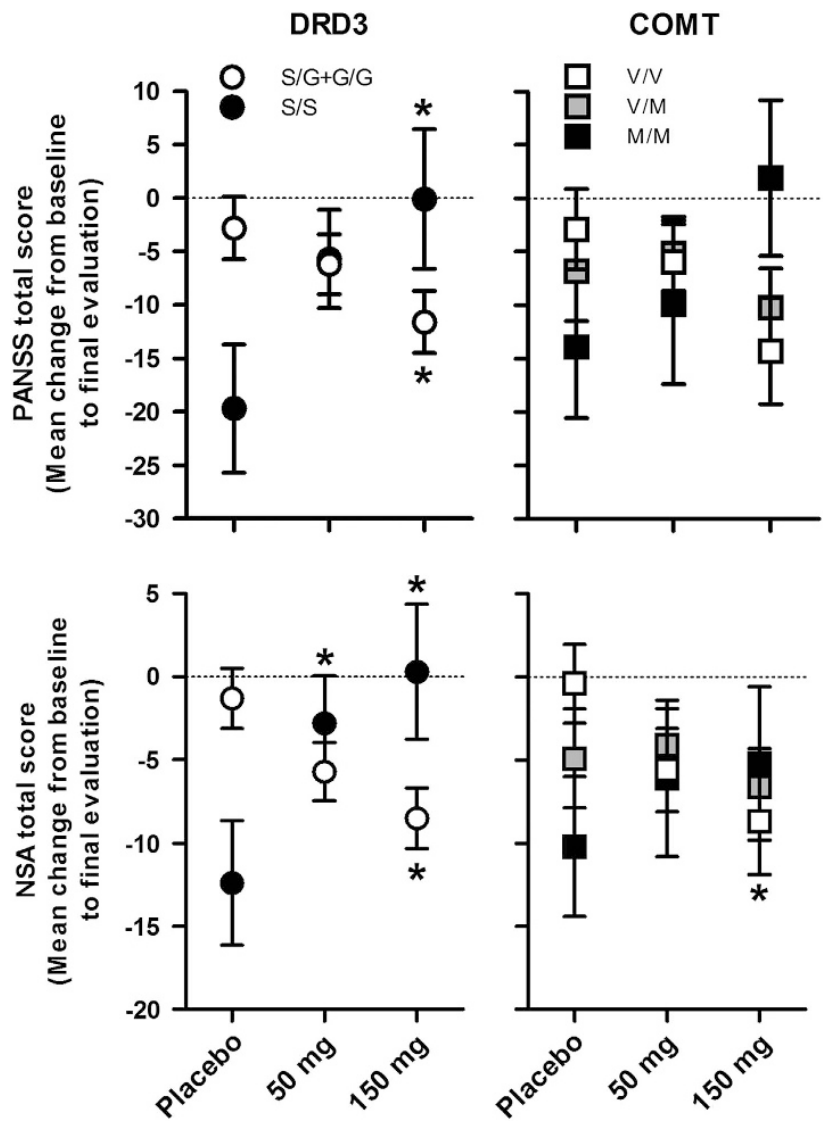

Figure 1 Dose-response relationship for ABT-925 on the Positive and Negative Syndrome Scale (PANSS; top) and Negative Symptom Assessment 16 (NSA-16; bottom) total scores for dopamine $\mathrm{D}_{3}$ receptor (DRD3; left) and the catechol-omethyl transferase (COMT; right) genotypes. Negative values represent improvement of symptoms at the final clinical evaluation compared with baseline. Error bars represent s.e. ${ }^{*} P<0.05$ compared with respective placebo group. n/group (placebo, $50 \mathrm{mg}, 150 \mathrm{mg}$ ): S/G + GG: 30, 32, 30; S/S: 7, 12, 6; V/V: 19, 17, 11; V/M: 12, 22, 20; M/M: 6, 5, 5 . 
sex or race between subjects who consented for pharmacogenetic analysis and all study subjects. Patient baseline characteristics for the intent-to-treat subjects have been previously published. ${ }^{2}$ The pharmacogenetic testing patient subset was $73 \%$ male; $30 \%$ white, $50 \%$ black and $20 \%$ other; with a mean (s.d.) age of 40.6 (9.0) years.

Genotypes. All genotypes were successfully determined and were in Hardy-Weinberg equilibrium. The DRD3 9G allele was observed at a frequency of 0.56 . The COMT V158 allele was observed at a frequency of 0.64 . Repeat length frequencies for the SLC6A3 VNTR were: 3 copies, 0.008; 7 copies, 0.008; 8 copies, 0.016; 9 copies, 0.211; 10 copies, 0.748 ; 11 copies, 0.004 and 12 copies, 0.004 .

Efficacy analyses. A DRD3 S9G genotype-by-treatment group interaction was observed for the mean change from baseline to the final evaluation for the PANSS total score $(P=0.015)$ and the NSA-16 total score $(P=0.004$; Figure 1$)$. Within the $S / G+G / G$ group, $150 \mathrm{mg} A B T-925$ produced improvements compared with placebo of 8.8 points on the PANSS total score and 7.2 points on the NSA-16. Within the $\mathrm{S} / \mathrm{S}$ group, this dose produced a worsening compared with placebo of 19.6 points on the PANSS total score, and both doses of ABT-925 produced a worsening on the NSA-16. For the COMT V158M locus, there was no statistically significant genotype-by-treatment group interaction for the PANSS total score $(P=0.219$; Figure 1$)$. The dose of $150 \mathrm{mg}$ ABT-925 produced an improvement of 8.3 points on the NSA-16 total score compared with placebo in subjects with the COMT V/V genotype; however, there was no statistically significant COMT V158M genotype-by-treatment group interaction. No significant effect of treatment was seen in the $\mathrm{V} / \mathrm{M}$ or

Table 1 Effects of genotype and ABT-925 treatment on PANSS subscale measurements

\begin{tabular}{|c|c|c|c|c|}
\hline & Genotype & Placebo & $50 \mathrm{mg} A B T-925$ & $150 \mathrm{mg} A B T-925$ \\
\hline \multicolumn{5}{|c|}{ PANSS_positive symptoms scale } \\
\hline \multirow[t]{2}{*}{ DRD3 } & $\mathrm{S} / \mathrm{G}+\mathrm{G} / \mathrm{G}$ & $-1.5(0.95)$ & $-2.0(0.92)$ & $-3.9(0.95)$ \\
\hline & $\mathrm{S} / \mathrm{S}$ & $-5.1(1.98)$ & $-2.9(1.51)$ & $-1.1(2.16)$ \\
\hline \multirow[t]{3}{*}{ COMT } & V/V & $-1.2(1.19)$ & $-1.8(1.25)$ & $-4.6(1.56)$ \\
\hline & $\mathrm{V} / \mathrm{M}$ & $-1.9(1.50)$ & $-2.2(1.11)$ & $-4.0(1.15)$ \\
\hline & $\mathrm{M} / \mathrm{M}$ & $-5.7(2.11)$ & $-3.7(2.32)$ & $1.4(2.31)^{*}$ \\
\hline \multicolumn{5}{|c|}{ PANSS-negative symptoms scale } \\
\hline \multirow[t]{2}{*}{ DRD3 } & $\mathrm{S} / \mathrm{G}+\mathrm{G} / \mathrm{G}$ & $-1.2(0.78)$ & $-2.1(0.76)$ & $-2.5(0.79)$ \\
\hline & $\mathrm{S} / \mathrm{S}$ & $-5.6(1.63)$ & $-1.2(1.24)^{\star}$ & $-0.4(1.75)^{*}$ \\
\hline \multirow[t]{3}{*}{ COMT } & $\mathrm{V} / \mathrm{V}$ & $-0.8(1.00)$ & $-1.9(1.06)$ & $-3.1(1.34)$ \\
\hline & $\mathrm{V} / \mathrm{M}$ & $-2.8(1.26)$ & $-1.8(0.93)$ & $-2.0(0.98)$ \\
\hline & $\mathrm{M} / \mathrm{M}$ & $-4.6(1.81)$ & $-2.4(1.99)$ & $-0.4(1.97)$ \\
\hline \multicolumn{5}{|c|}{ PANSS_-general psychopathology scale } \\
\hline \multirow[t]{2}{*}{ DRD3 } & $\mathrm{S} / \mathrm{G}+\mathrm{G} / \mathrm{G}$ & $-0.3(1.49)$ & $-2.2(1.44)$ & $-5.1(1.49)^{*}$ \\
\hline & $\mathrm{S} / \mathrm{S}$ & $-9.0(3.08)$ & $-1.6(2.35)$ & $1.9(3.34)^{*}$ \\
\hline \multirow[t]{3}{*}{ COMT } & $\mathrm{V} / \mathrm{V}$ & $-1.2(1.95)$ & $-2.4(2.05)$ & $-6.2(2.56)$ \\
\hline & $\mathrm{V} / \mathrm{M}$ & $-2.5(2.45)$ & $-1.0(1.81)$ & $-3.9(1.89)$ \\
\hline & $\mathrm{M} / \mathrm{M}$ & $-3.3(3.46)$ & $-5.5(3.85)$ & $1.1(3.79)$ \\
\hline
\end{tabular}

Abbreviations: DRD3, dopamine D3 receptor; COMT, catechol-o-methyl transferase; PANSS, Positive and Negative Syndrome Scale.

Values represent the model-based mean (s.e.) change from baseline to the final evaluation on each subscale. Negative values represent improvement in symptoms compared with baseline assessment. ${ }^{\star} P<0.05$ versus placebo. n/group (placebo, 50 mg, $150 \mathrm{mg}$ ): S/G + GG: 30, 32, 30; S/S: 7, 12, 6; V/V: 19, 17, 11; V/M: 12, 22, 20; M/M: 6, 5, 5 .
M/M genotype groups. Similar trends were seen across loci and genotypes on each PANSS subscale (see Table 1). There were no significant effects detected in analyses of SLC6A3 genotypes (data not shown).

\section{Discussion}

The therapeutic efficacy of D3 antagonism in schizophrenia has not yet been confirmed. A minimum receptor occupancy may be required for D3 antagonism to produce antipsychotic effects, as has been shown for the D2 receptor. ${ }^{9,10}$ A follow-up study on 50 and $150 \mathrm{mg}$ regimens of ABT-925 revealed $<60 \%$ receptor occupancy at the D3 receptor, ${ }^{3}$ which may have limited the clinical efficacy of these doses. Nonetheless, our results coupled with the reported increased D3 receptor binding affinity for dopamine and D3 antagonists resulting from the DRD 3 S9G polymorphism, ${ }^{4}$ suggest that possessing at least one $G$ allele may confer a wider therapeutic window for D3 antagonists. Similarly, subjects with increased dopamine tone resulting from expression of the COMT V158M polymorphism $^{11}$ may be less likely to respond to D3 receptor antagonists.

Given the size of the data set, we cannot eliminate the possibility that the observed clinical significance of these markers is surrogate for factors which were not analyzed (for example, ethnicity). In addition, the current findings might not apply to patients not represented in the original clinical trial, such as treatment-resistant schizophrenia. Although sample sizes of the S/S and M/M genotype groups were relatively small, and the regimens did not provide sufficient receptor occupancy to assess clinical significance, these results support the notion that pharmacogenetic analyses may reveal sub-populations of schizophrenia patients who could respond favorably to D3 receptor antagonists. However, higher D3 receptor occupancy by a D3 antagonist should be achieved in order to fully understand therapeutic efficacy in schizophrenia and the effects of DRD3 polymorphisms. Thus, further research in this area is warranted.

\section{Conflict of interest}

All authors were employees of AbbVie at the time the studies were conducted. AbbVie participated in the study design, research, data collection, analysis and interpretation of data, writing, review and approving the publication. Authors Bhathena, Wang, Idler, Robieson and Katz are employees of AbbVie; receive salary and other compensation from AbbVie; hold AbbVie stock and/or stock options. Ms Holley-Shanks is an employee of AbbVie; receives salary and other compensation from AbbVie. Drs Kraft, Redden and Spear are former employees of AbbVie; hold AbbVie stock and/or stock options. Dr Kraft is currently employed by the United States Food and Drug Administration, Silver Springs, MD, USA. Dr Redden is currently employed by Advanced Clinical, Deerfield, IL, USA. Dr Katz is an AbbVie patent holder.

Acknowledgements. This study was funded by AbbVie Inc., and AbbVie provided the study drug (ABT-925). We thank the site investigators and coordinators for their efforts in the conduct of the study. Nathan R Rustay and Michelle M Tangredi, employees of AbbVie, provided medical writing support for the manuscript. 
1. Geneste H, Amberg W, Backfisch G, Beyerbach A, Braje WM, Delzer J et al. Synthesis and SAR of highly potent and selective dopamine D3-receptor antagonists: variations on the 1H-pyrimidin-2-one theme. Bioorg Med Chem Lett 2006; 16: 1934-1937.

2. Redden L, Rendenbach-Mueller B, Abi-Saab WM, Katz DA, Goenjian A, Robieson WZ et al. A double-blind, randomized, placebo-controlled study of the dopamine D receptor antagonist ABT-925 in patients with acute schizophrenia J Clin Psychopharmacol 2011; 31: 221-225.

3. Graff-Guerrero A, Redden L, Abi-Saab W, Katz DA, Houle S, Barsoum P et al. Blockade of $[11 \mathrm{C}](+)$-PHNO binding in human subjects by the dopamine D3 receptor antagonist ABT-925. Int J Neuropsychopharmacol 2010; 13: 273-287.

4. Lundstrom K, Turpin MP. Proposed schizophrenia-related gene polymorphism: expression of the Ser9Gly mutant human dopamine D3 receptor with the Semliki Forest virus system. Biochem Biophys Res Commun 1996; 225: 1068-1072.

5. Arranz MJ, de Leon J. Pharmacogenetics and pharmacogenomics of schizophrenia: a review of last decade of research. Mol Psychiatry 2007; 12: 707-747.

6. Bertolino A, Caforio G, Blasi G, Rampino A, Nardini M, Weinberger DR et al. COMT Val158Met polymorphism predicts negative symptoms response to treatment with olanzapine in schizophrenia. Schizophr Res 2007; 95: 253-255.

7. Molero P, Ortuno F, Zalacain M, Patino-Garcia A. Clinical involvement of catechol-Omethyltransferase polymorphisms in schizophrenia spectrum disorders: influence on the severity of psychotic symptoms and on the response to neuroleptic treatment. Pharmacogenomics J 2007; 7: 418-426.
8. van Dyck CH, Malison RT, Jacobsen LK, Seibyl JP, Staley JK, Laruelle M et al. Increased dopamine transporter availability associated with the 9-repeat allele of the SLC6A3 gene. J Nucl Med 2005; 46: 745-751.

9. Farde L, Nordstrom AL, Wiesel FA, Pauli S, Halldin C, Sedvall G. Positron emission tomographic analysis of central D1 and D2 dopamine receptor occupancy in patients treated with classical neuroleptics and clozapine. Relation to extrapyramidal side effects. Arch Gen Psychiatry 1992; 49: 538-544.

10. Kapur S, Zipursky RB, Remington G. Clinical and theoretical implications of 5-HT2 and D2 receptor occupancy of clozapine, risperidone, and olanzapine in schizophrenia. Am J Psychiatry 1999; 156: 286-293.

11. Tunbridge EM, Harrison PJ, Weinberger DR. Catechol-o-methyltransferase, cognition, and psychosis: Val158Met and beyond. Biol Psychiatry 2006; 60: $141-151$.

(i) $($ ) $\ominus$ Translational Psychiatry is an open-access journal published by Nature Publishing Group. This work is licensed under a Creative Commons Attribution-NonCommercialNoDerivs 3.0 Unported License. To view a copy of this license, visit http://creativecommons.org/licenses/by-nc-nd/3.0/ 